\title{
Prévision et gestion des crues du Rhône supérieur par l'exploitation optimale des retenues alpines
}

\author{
Flood forecast and management model of the Rhone river : optimal use of the \\ alpine hydropower schemes for the reduction of peak flows \\ FRÉDÉRIC JORDAN \\ e-dric.ch eau énergie environnement ingénieurs conseils, Ch. du Rionzi 54, CH-1052 Le Mont-sur-Lausanne, Suisse \\ Tel : ++41 2178433 13, Fax : ++41 2178433 22, e-mail : fred.jordan@e-dric.ch
}

JEAN-LOUIS BOILLAT, ANTON SCHLEISS

Laboratoire de constructions hydrauliques (LCH), Ecole polytechnique fédérale de Lausanne (EPFL), Station 18, CH-1015 Lausanne, Suisse

Tel : ++41 2169323 85, Fax : ++41 2169322 64, e-mail : secretariat.lch@epfl.ch

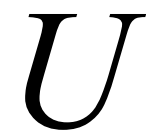

$n$ modèle de prévision et de gestion des crues du Rhône supérieur à l'amont du Léman est présenté. Cet outil permet la prévision des débits à un horizon de $72 \mathrm{~h}$ en tout point du bassin versant et consécutivement une gestion préventive optimale des aménagements hydroélectriques à accumulation lors des crues. Tenant compte des différents processus hydrologiques présents sur le bassin versant, le modèle est également capable d'améliorer sa pertinence grâce à une procédure de mise à jour des variables d'état. Enfin, un système d'aide à la décision permet, pour chaque retenue, de déterminer les horaires optimaux de turbinage et d'ouverture des vidanges de fond pour maximiser l'effet de laminage lors de la pointe de la crue. Il produit également une estimation du coût associé aux décisions ou non-décisions ainsi que le coût de décisions inappropriées résultant d'une fausse prévision météorologique.

$A$ semi-distributed hydrological model was developed for the upper Rhone River basin in Switzerland to provide $72 \mathrm{~h}$ lead time discharge forecasts for the optimization of a multireservoir system during floods. The performance of the model is presented and correlated with the spatial distribution of the hydrological processes. Moreover, a methodology for the data assimilation by the model is presented and its performance for decision-making is confirmed. A decision-making tool was developed, which is able to optimize the preventive operations of multiple existing hydropower plants with large reservoirs in order to limit the flood damages in the basin. It allows the decision maker to estimate the cost of the decisions and of the non-decisions, as well as of the cost of an inappropriate decision due to false flood forecast.

\section{I INTRODUCTION}

Le bassin versant du Rhône à l'amont du lac Léman se situe en territoire suisse sur les cantons du Valais et de Vaud. Il s'étend sur une surface de $5520 \mathrm{~km}^{2}$, culmine à 4634 m s.m. à la Pointe Dufour et son exutoire dans le Léman se trouve à l'altitude de $372.50 \mathrm{~m}$ s.m. Une particularité de ce bassin versant, outre son comportement hydrologique glacio-nival, réside dans la présence de nombreux aménagements hydroélectriques d'importance nationale, constitués de grandes retenues ayant un volume utile total de $1200 \mathrm{Mm}^{3}$. Ces dernières présentent un potentiel intéressant pour la gestion des crues grâce à leur capacité de stockage du volume ruisselé. Cette faculté est accentuée par les nom- breuses installations de captage et de pompage des eaux de bassins versants éloignés des retenues principales, dont les surfaces drainées représentent près de $30 \%$ de la surface totale du bassin versant (fig. 1).

Le bassin versant du Rhône à l'amont du Léman constitue une vallée intra-alpine qui se situe directement au Nord de la crête principale des Alpes pennines, dans la région du Valais. Il est de ce fait relativement bien protégé des perturbations provenant du Nord-Est jusqu'au Nord-Ouest en passant par le Nord, mais particulièrement vulnérable aux perturbations provenant du Sud et du Sud-Est qui provoquent des effets de barrage orographique. Ainsi, lors des 20 dernières années, le bassin versant du Rhône supérieur a subi trois crues majeures $(1987,1993,2000)$ provoquées par d'intenses précipi- 
Surface bassin versant : $5520 \mathrm{~km}^{2}$

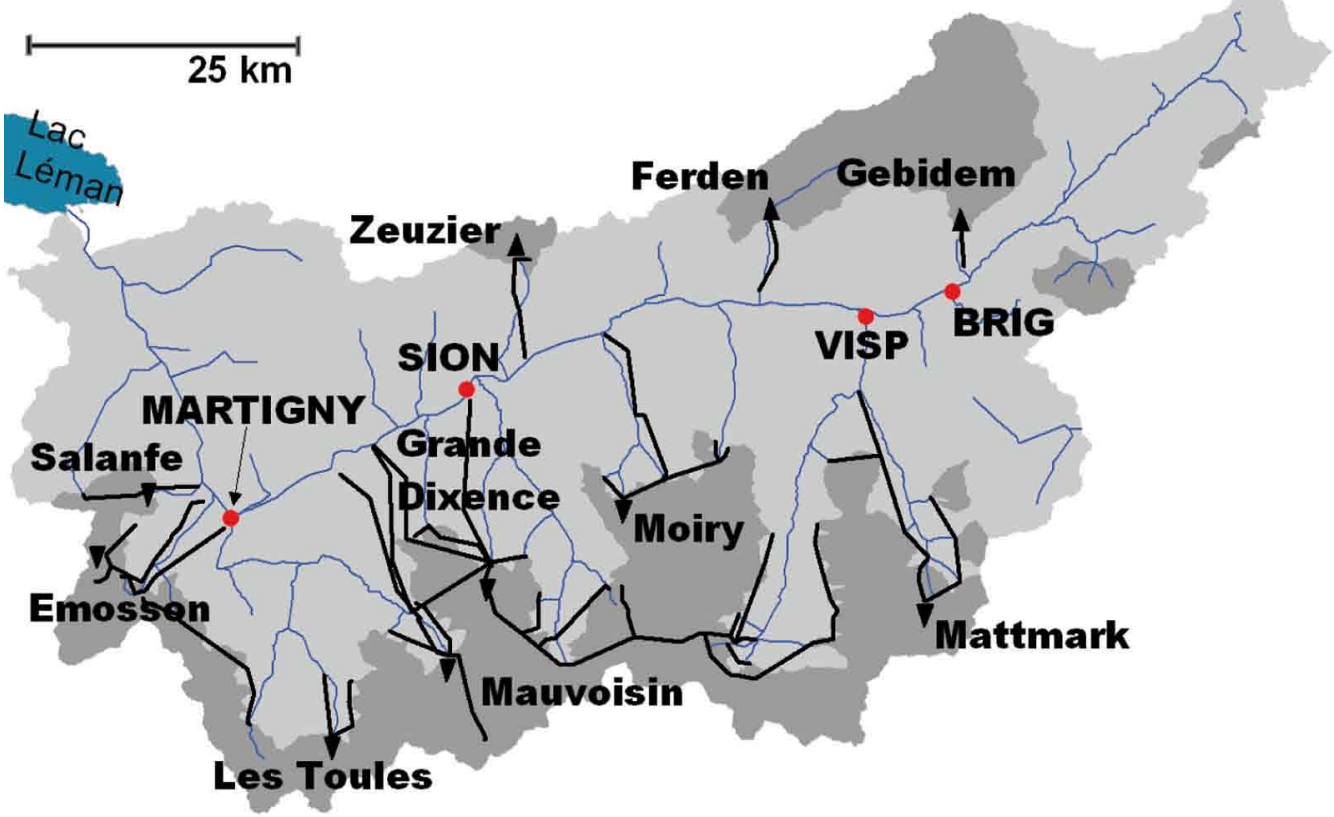

Figure 1 : Carte du bassin versant du Rhône supérieur avec les principaux aménagements à accumulation. Les surfaces grisées représentent les surfaces drainées vers les retenues alpines.

tations durant plusieurs jours, issues de courants humides arrivant du Sud-Est et de la mer Adriatique. Ces crues localement d'ordre centennal ont provoqué d'importants dégâts aux biens et aux personnes, notamment en raison de débordements du Rhône, mais aussi de phénomènes de dépôt de matériaux solides, d'éboulements et de laves torrentielles associées aux fortes précipitations.

Lors de ces récentes crues, les retenues alpines ont toutefois joué un important rôle protecteur (Raboud et al., 2001, Boillat, 2005, Jordan et al., 2006). En effet, les grands aménagements hydroélectriques ont globalement davantage stocké que libéré les volumes entrant et captés, et cela malgré un niveau de remplissage élevé au début des crues (Jordan, 2005). Il convient de noter que ces retenues fonctionnement en général selon un cycle d'accumulation annuel et qu'elles sont pratiquement pleines depuis fin août jusqu'à mi-octobre, lorsque se produisent en général les fortes précipitations.

Ces différentes raisons ont conduit les autorités valaisannes à se doter d'un nouvel outil de prévision de débit et de gestion des aménagements hydroélectriques lors des crues. Le but de ce système appelé MINERVE, acronyme qui signifie « Modélisation des Intempéries de Nature Extrême, des Retenues Valaisannes et de leurs Effets », est d'optimiser l'influence protectrice des aménagements hydroélectriques lors des crues du Rhône. Par la suite, le fonctionnement et les différents composants de MINERVE seront décrits en détail, notamment le concept général du système, le modèle de prévision hydrologique semi-distribué, le modèle d'aide à la décision ainsi que la performance attendue et opérationnelle du système complet.

\section{II — CONCEPT GENERAL DE FONCTIONNEMENT DU SYSTEME MINERVE}

Le schéma de fonctionnement du système MINERVE est présenté dans la figure 2. Les informations provenant des différents fournisseurs sont stockées dans une base de données. Il s'agit en particulier des prévisions météorologiques issues du modèle aLMo de MétéoSuisse (Kaufmann et al., 2003), des mesures en temps réel des précipitations, températures et débits, et enfin des données provenant des aménagements hydroélectriques relatives aux niveaux des retenues et au fonctionnement des différents éléments (captages, turbines, pompes, ouvrages d'évacuation).

Ces données sont ensuite récupérées par le modèle de prévision hydrologique. Tout d'abord, les mesures de précipitation et de température sont utilisées pour effectuer des simulations hydrologiques relatives aux 24 dernières heures. Les résultats obtenus sont comparés aux mesures de débit aux différents points de contrôle du modèle. Cette procédure, appelé filtrage, permet de corriger les variables d'état du modèle hydrologique jusqu'à ce que les résultats issus de la simulation correspondent aux observations de débit. Les nouvelles conditions initiales sont alors implémentées et, par l'introduction des prévisions de précipitation et de température, une prévision de débit est effectuée en tout point du modèle hydrologique. Actuellement, l'horizon de prévision est de 72 heures à pas de temps horaire.

Les résultats de la prévision hydrologique sont ensuite importés dans le modèle d'aide à la décision pour la ges- 


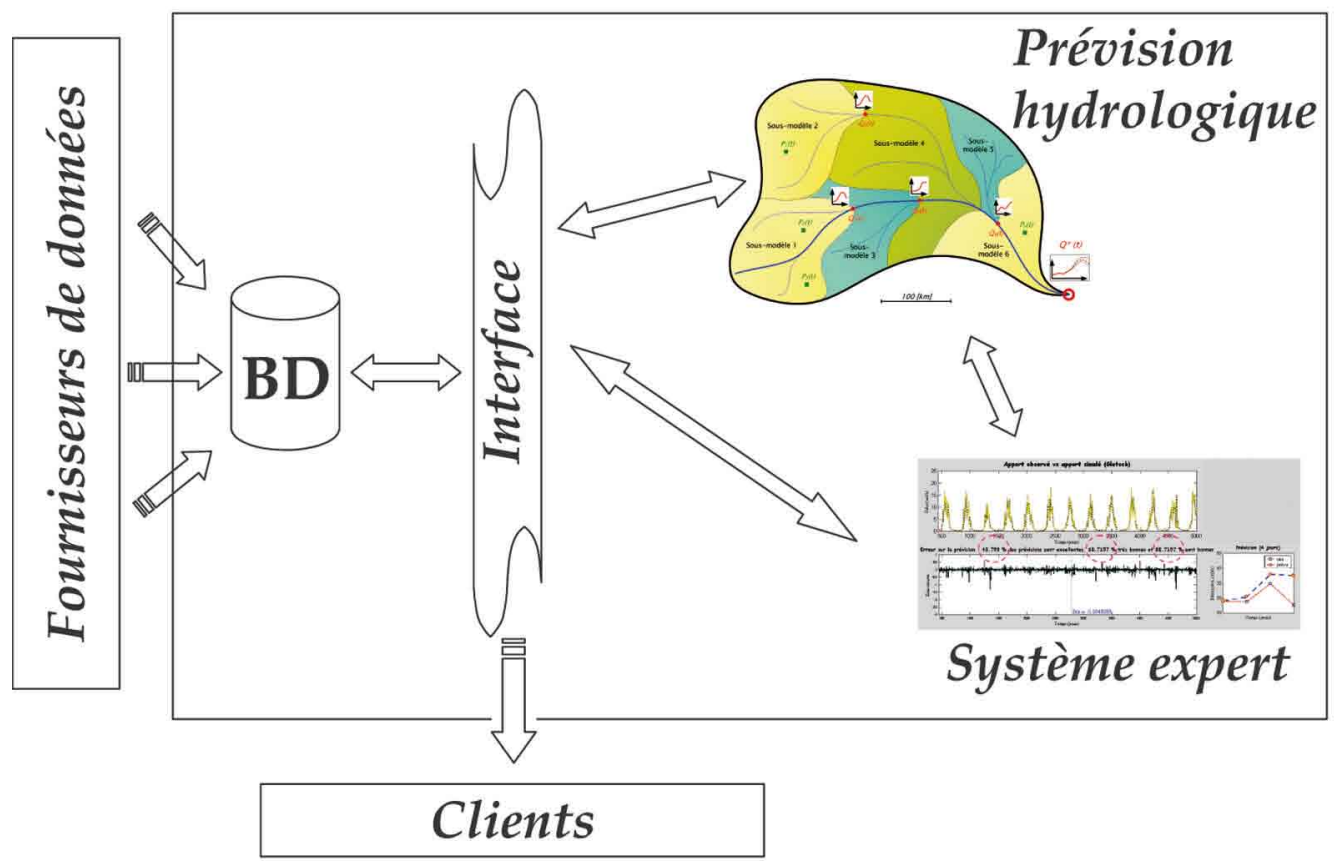

Figure 2 : Schéma de fonctionnement à haut niveau du système MINERVE

tion des aménagements hydroélectriques. Les débits prévus à l'entrée des retenues ainsi que les débits aux points de contrôle du modèle sont utilisés pour déterminer les opérations de vidange préventive optimales des retenues à accumulation. Les résultats issus de cette procédure sont les horaires de turbinage préventif et si nécessaire d'ouverture des vidanges de fond pour les prochaines 72 heures des 10 principaux aménagements. Les coûts et gains associés à ces opérations sont également calculés.

Finalement, les résultats de l'optimisation sont automatiquement renvoyés au modèle de simulation hydrologique pour validation. Si les hydrogrammes prévus aux points de contrôle suite aux opérations préventives correspondent aux hydrogrammes prévus par le système expert, la solution est validée. A cet instant, les responsables de la sécurité civile ont la possibilité d'exiger des exploitants d'aménagements hydroélectriques qu'ils procèdent à des opérations de vidange préventive. En cas de manœuvres inappropriées, ces derniers pourront le cas échéant exiger des compensations financières pour pertes d'eau et perte de flexibilité dans le processus de vente d'énergie.

\section{MODELE DE PREVISION HYDROLOGIQUE}

\section{III.1 CONCEPT DE MODÉLISATION}

Le modèle de prévision hydrologique est un modèle semidistribué : il s'agit d'un modèle conceptuel dont la résolution spatiale est relativement fine mais irrégulière. Ce concept permet l'introduction aisée des ouvrages hydrauliques dans la modélisation et la prise en compte des différents processus hydrologiques dans la région où ils se produisent. Le modèle hydrologique complet du bassin versant du Rhône à l'amont du Léman, d'une surface totale de $5520 \mathrm{~km}^{2}$, est découpé en 239 sous-bassins versants de $23 \mathrm{~km}^{2}$ de surface moyenne. Ce découpage ne permet cependant pas la prise en compte optimale des processus liés à la température, comme l'accumulation et la fonte de neige ainsi que la fonte de glace. Pour cette raison, les sous-bassins versants ont été eux-mêmes divisés en bandes d'altitude d'une dénivellation moyenne de $500 \mathrm{~m}$. Au final, le modèle se compose de 1054 bandes d'altitude, qui sont référencées par les coordonnées $\mathrm{X}, \mathrm{Y}$ et $\mathrm{Z}$ de leur centre de gravité ainsi que par leur surface.

Pour représenter le plus fidèlement possible les processus d'accumulation et de fonte de neige, de fonte de glace, d'infiltration et de ruissellement de surface, différents types de modèles de réservoirs ont été développés, qui permettent le fonctionnement du système MINERVE en simulation continue (Hamdi et al., 2005). Cela signifie que le modèle n'est pas uniquement apte à reproduire des événements particuliers, mais qu'il résiste à une simulation multi-annuelle en continu. Les différentes variables produisent donc des cycles journaliers, saisonniers ou annuels selon leurs particularités, en n'étant initialisées qu'une seule fois au début de la simulation.

Le concept hydrologique se base sur le modèle SOCONT (Consuegra et al., 1998) développé à l'HYDRAM (Laboratoire d'hydrologie et aménagements de l'Ecole Polytechnique Fédérale de Lausanne). Il a été étendu aux processus de fonte nivale et glaciaire (Schäfli, 2005, Jordan, 2007, 
Garcia et al., 2007) et se nomme maintenant GSM-SOCONT (Glacier Snow Melt - Soil CONTribution model). Selon ce concept de modélisation semi-distribuée, les données d'entrée (variables météorologiques) sont des précipitations, des températures et des valeurs d'évapo-transpiration potentielle (ETP) alors que les variables de sortie sont des débits. Chaque bande d'altitude est une entité hydrologique avec ses entrées et sorties propres, qui peut être soit glaciaire soit non-glaciaire (fig. 3).

Dans la situation glaciaire, un modèle de fonte de neige (Neige) reçoit en entrée les variables météorologiques de précipitation $P$ et de température $T$. Il est connecté en série à un modèle de fonte glaciaire (Glacier). La pluie équivalente $P_{e q}$ produite par la fonte de neige est transférée au réservoir linéaire de neige $R N$ qui produit un débit de fonte de neige $Q_{N G L}$. Si la hauteur de neige $H_{N}$ sur le glacier est nulle, le modèle de fonte de glace produit une pluie équivalente glaciaire $P_{e q G L}$ acheminée vers le réservoir linéaire de glace $R G L$. Ce dernier fournit le débit de fonte glaciaire $Q_{G L}$

Dans la situation non-glaciaire, le modèle de fonte de neige transfère la pluie équivalente $P_{e q}$ au réservoir-sol ( $\mathrm{Sol}$, modèle GR3). Ce dernier accepte également en entrée l'ETP et fournit à l'exutoire le débit $Q_{s}$, somme d'une composante d'infiltration et de ruissellement de surface (modèle SWMM). Finalement, le débit à l'exutoire de la bande d'altitude $Q_{\text {tot }}$ est la somme de $Q_{s}, Q_{N G L}$ et $Q_{G L}$

Le fonctionnement du modèle hydrologique est présenté à la figure 4 pour un bassin versant fortement glaciaire (bassin versant du glacier d'Aletsch, surface représentant $54 \%$ de la surface totale du sous-bassin versant de $198.5 \mathrm{~km}^{2}$ ). Il est possible de discerner la composante débit de base produite tout au long de l'année, la fonte de neige entre avril et juillet, la fonte glaciaire de juillet à septembre et enfin la crue générée par d'intenses précipitations en octobre 2000. Ces simulations sont finalement comparées aux observations pour s'assurer de la pertinence de la modélisation.

Les performances du modèle hydrologique sont variables en fonction des régions du bassin versant du Rhône et des processus hydrologiques s'y produisant. Il est dès lors intéressant d'analyser le comportement du modèle hydrologique à travers une vision spatiale de la performance de la simulation (fig. 5). Cette figure présente une carte du bassin versant du Rhône supérieur avec en couleur les valeurs obtenues du critère de Nash (Nash \& Sutcliffe, 1970). Les valeurs proches de 1 sont optimales, tandis que des valeurs inférieures à 0.5 dénotent d'une qualité de modélisation plus médiocre.

Comme observé sur la figure 5, le modèle hydrologique reproduit fidèlement les processus hydrologiques, non seulement dans la vallée principale du Rhône mais également dans les sous-bassins versants de tête, en particulier ceux situés à l'amont des retenues alpines. Par contre, le modèle rencontre quelques difficultés lors du calcul du débit dans les vallées latérales situées au Sud du bassin versant. Il s'agit principalement de la vallée de la Viège (Sud-Est), à l'amont de laquelle se trouve la retenue de Mattmark et des vallées des Dranses comportant les barrages de Mauvoisin et des Toules. Dans ces sous-bassins versants, les eaux provenant de l'amont et donc des régions de haute altitude sont toutes captées et acheminées vers les retenues hydroélectriques. Il

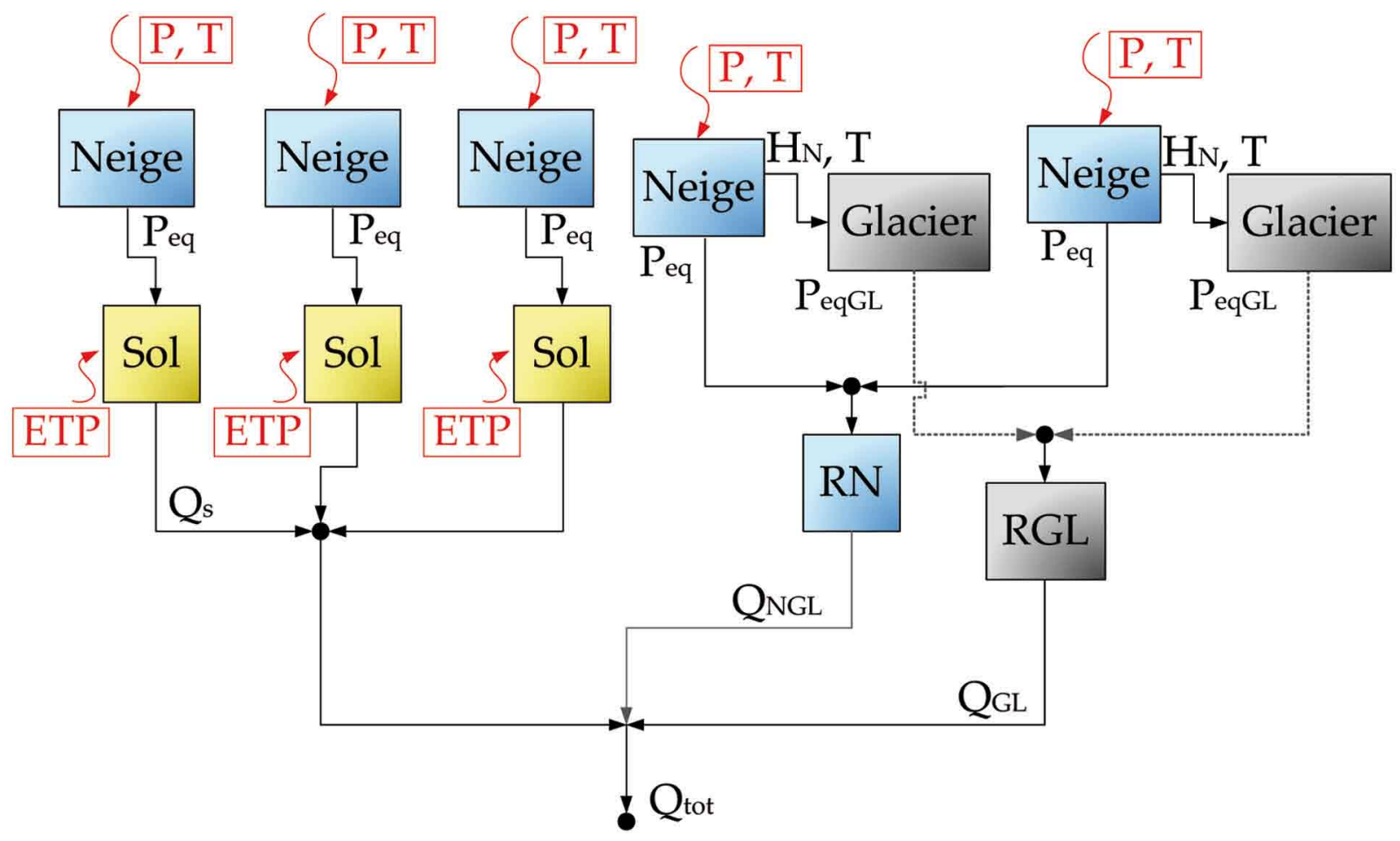

Figure 3 : Schéma du modèle hydrologique semi-distribué avec trois modèles en réservoirs (sol, neige, glacier) 


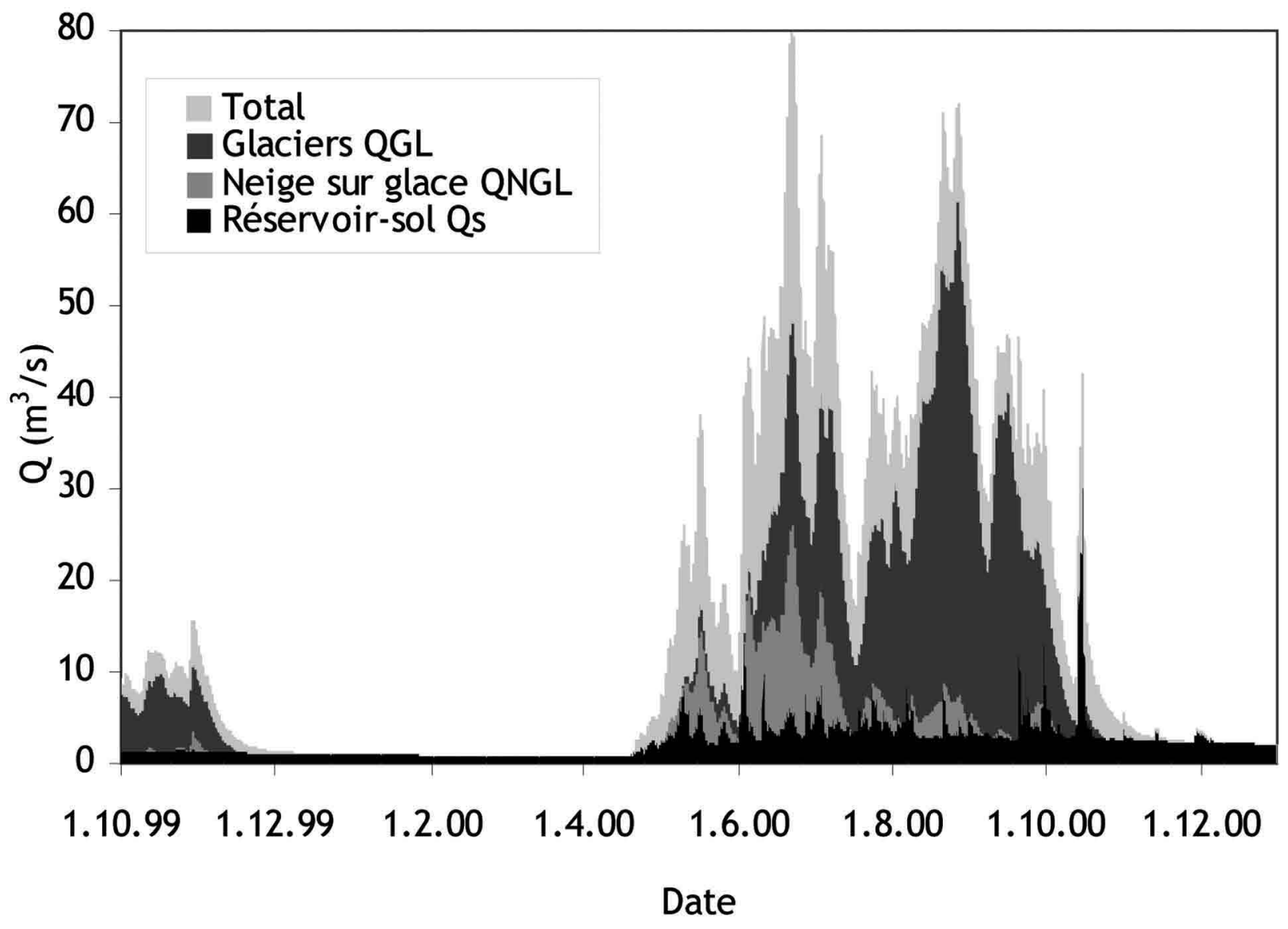

Figure 4 : Fonctionnement du modèle hydrologique, exemple du bassin versant du glacier d'Aletsch.

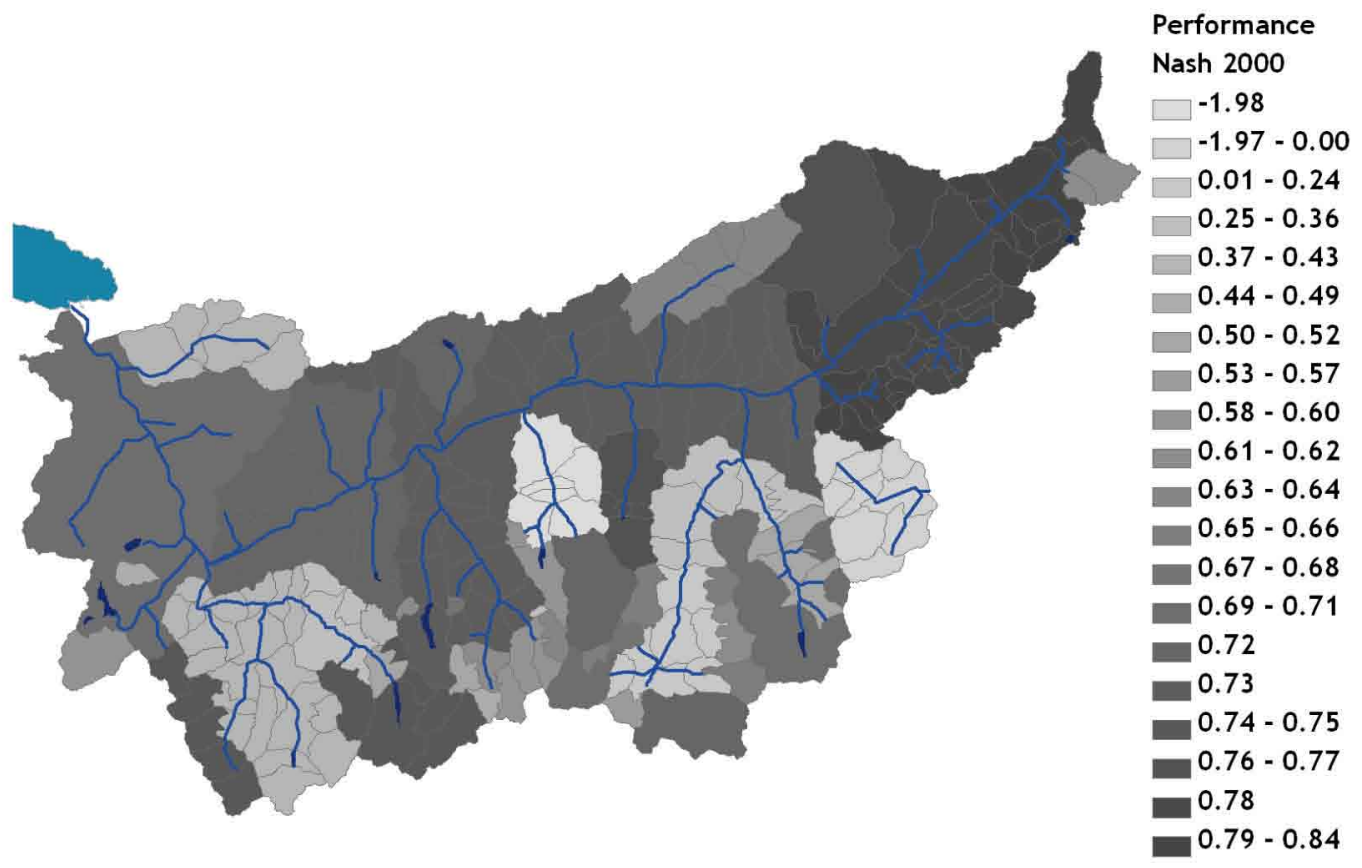

Figure 5 : Carte des performances exprimées par le coefficient de Nash du modèle hydrologique dans les différents sousbassins versants. 
ne reste dans les vallées Sud que les eaux drainées par les flancs situés à l'aval des retenues. Ces derniers sont composés de pentes raides et rocailleuses ou couvertes de forêts de conifères, abritées par les hauts sommets avoisinants. Il s'agit donc de conditions difficiles pour la modélisation, car d'une part les données météorologiques sont très imprécises (les stations de mesure sont peu nombreuses et situées aux extrémités amont et aval des vallées) et les phénomènes météorologiques locaux (notamment certains effets orographiques) ne peuvent pas être pris en compte. Enfin, les processus hydrologiques dans ces régions sont vraisemblablement plus complexes que ceux considérés par le modèle et nécessitent une analyse complémentaire associée à une densification du réseau de mesures.

La performance globale du modèle hydrologique peut être évaluée par la comparaison entre les simulations et les observations à l'exutoire du bassin versant du Rhône dans le Léman. L'année 2000 est présentée à titre d'exemple à la fig. 6, année au cours de laquelle la plus grande crue observée jusqu'ici dans ce bassin versant s'est déroulée. Le coefficient de Nash obtenu est de 0.71 et la corrélation de 0.85 . Le cycle hydrologique annuel y est très bien représenté, avec la saison d'hiver où le débit est soumis au marnage des aménagements à accumulation et à son cycle journalier et hebdomadaire. La fonte de neige débute vers la mi-avril, suivie de la fonte des glaciers. Finalement, la crue d'octobre 2000 a parfaitement été reproduite par le modèle hydrologique, comme l'atteste le graphe de corrélation entre débits observés et simulés.

$\mathrm{Au}$ final, les informations les plus importantes pour la prise de décision sont tout de même disponibles avec une fiabilité suffisante. En effet, les débits entrant dans les rete- nues sont en général bien estimés par le modèle hydrologique, ce qui permet de prévoir les opérations préventives par comparaison avec le volume de stockage disponible au début de l'événement. De plus, les débits aux points de contrôle, tous situés dans la vallée principale, sont également estimés avec une précision suffisante pour permettre une évaluation réaliste de l'influence des opérations préventives.

\section{III.2 PROCÉDURE DE MISE À JOUR DU MODÈLE HYDROLOGIQUE}

Une procédure de mise à jour du modèle hydrologique a été développée, afin de garantir que le modèle produise des résultats conformes aux observations avant de démarrer une prévision (Jordan, 2007). Parmi les nombreuses méthodes de filtrage et d'ajustement en temps réel, une procédure relativement simple a été développée. Il s'agit non pas de modifier les variables d'entrée du modèle (précipitation, température) ou les paramètres du modèle hydrologique, mais plutôt d'ajuster les stocks des nombreux réservoirs composant le modèle hydrologique pour lui permettre de corriger le débit des sous-bassins versants. Il s'agit donc d'une procédure de mise à jour des variables d'état du modèle.

Les hypothèses admises pour le développement de la procédure sont les suivantes :

Le modèle est « bon » : le rapport entre les différentes composantes de débit à l'amont d'un point de contrôle peut être conservé. En pratique, cela signifie que tous les niveaux des réservoirs hydrologiques des différents sous-bassins versants sont modifiés dans la même proportion.

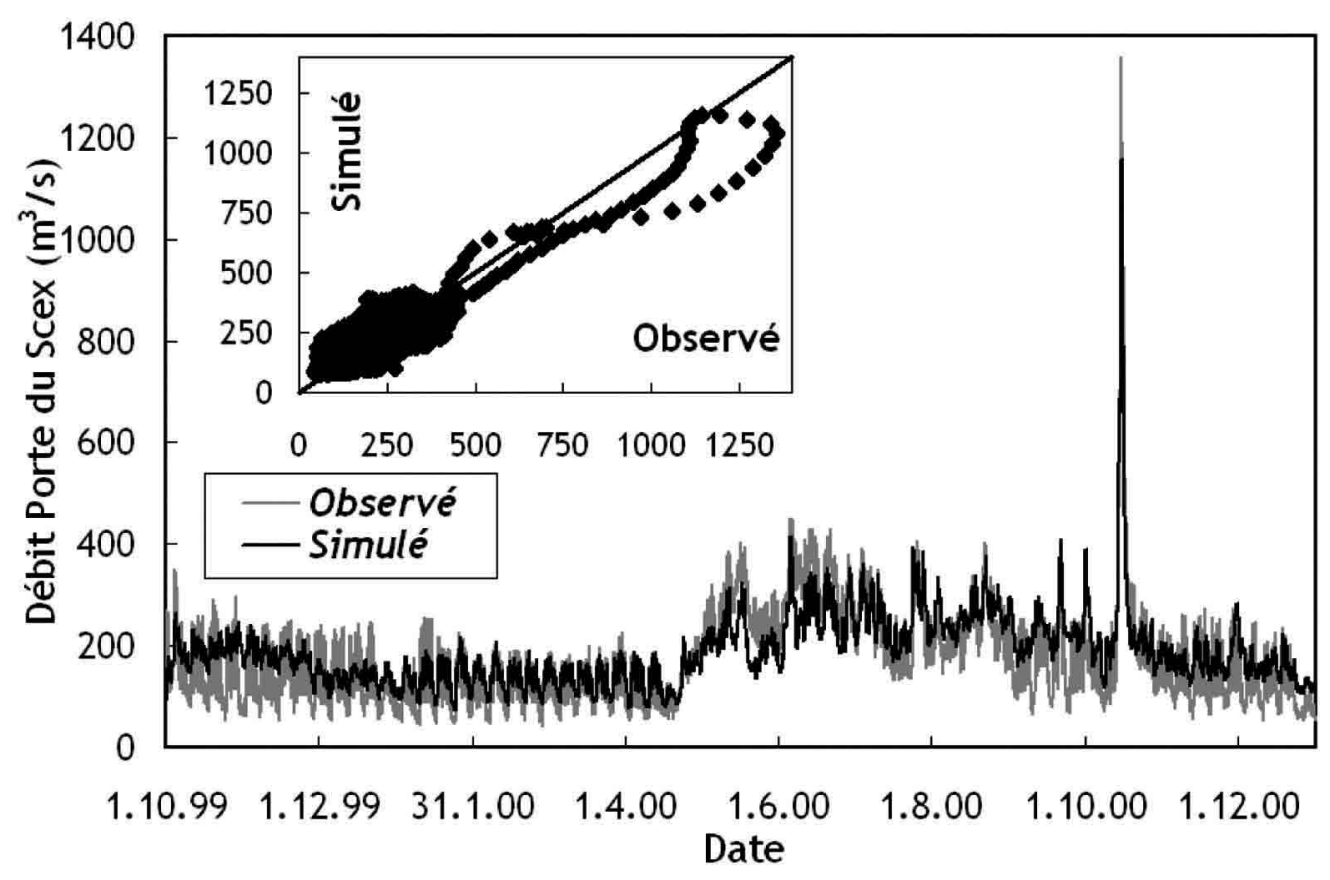

Figure 6 : Comparaison entre débits simulés et observés à la Porte du Scex (exutoire du bassin versant du Rhône supérieur dans le Léman). 
Il n'y a pas de préférence dans le choix du processus hydrologique à corriger : les niveaux des réservoirs linéaires de glace et de neige ainsi que des réservoirs GR3 et SWMM sont ajustés.

Une période d'analyse des volumes minimale de 12 heures doit permettre de négliger l'effet du temps de transfert de l'onde de crue de l'amont vers l'aval sur le secteur considéré. Cette durée permet également de réduire les incertitudes dues au turbinage.

Le temps de transfert d'une zone à l'autre peut être négligé : si l'ajustement du volume d'une zone située à l'amont est bon, le débit simulé peut être directement introduit dans le modèle et non la mesure. Il n'y a dès lors pas de propagation de l'incertitude des zones amont vers les zones aval.

Le déroulement de l'algorithme de mise à jour suit plusieurs étapes. En outre, plusieurs zones peuvent être filtrées en parallèle, pour autant qu'elles n'aient pas d'influence les unes sur les autres. Ainsi, les sous-bassins versants de tête peuvent être filtrés simultanément (zones de niveau 1), puis les sous-bassins intermédiaires (zones de niveau 2), et ainsi de suite jusqu'à l'exutoire du bassin versant.

Il s'agit donc d'un algorithme à double boucle. La première boucle concerne les niveaux de zone et ne fait pas l'objet d'itération. La deuxième boucle est une itération sur le calcul du coefficient de correction avec pour critère d'acceptation la convergence de la solution ou le nombre d'itérations. Ce coefficient de correction $C_{M A J}$ peut se calculer selon la formule suivante (équ. 1):

$$
C_{M A J}=1+\eta \cdot \frac{V_{o b s}-V_{s i m}}{V_{s i m}}
$$

où $\eta$ est un facteur de convergence fixé à une valeur entre 0.90 et $0.95, V_{o b s}$ et $V_{\text {sim }}$ le volume observé, respectivement simulé pendant la période de mise à jour.

Une fois le coefficient de correction calculé à l'itération $n$, la nouvelle condition initiale $h_{i n i}$ d'un réservoir du modèle pour l'itération suivante s'obtient facilement (équ. 2) :

$$
h_{i n i, n+1}=C_{M A J} \cdot h_{i n i, n}
$$

Les 4 réservoirs du modèle hydrologique sont corrigés simultanément afin de ne pas discriminer un processus hydrologique au détriment des autres. La correction d'une partie seulement des réservoirs résulterait d'une hypothèse sur la source de l'erreur, qui n'est toutefois pas vérifiable. En effet, le débit observé, utilisé comme mesure de contrôle, ne permet pas de séparer les différents processus de formation de l'écoulement. Il serait ainsi dangereux de prétendre qu'un déficit en eau du modèle lors d'une crue provient plus de la fonte que du ruissellement de surface (donc de la précipitation). L'utilisation systématique de la procédure de mise à jour permet d'améliorer localement la performance $\mathrm{du}$ modèle hydrologique mais ne constitue en aucun cas une solution pour compenser certains déficits structurels ou fonctionnels du modèle.

\section{MODELE D'AIDE A LA DECISION}

\section{IV.1 OBJECTIFS, CONTRAINTES ET VARIABLES D'OPTIMISATION}

Lors des crues, l'objectif prioritaire des responsables de la sécurité civile est de limiter les pertes humaines et les dégâts matériels. Dans le cas du bassin versant du Rhône à l'amont du Léman, les nombreux aménagements hydroélectriques représentent une chance à cet égard car ils constituent un levier efficace pour le contrôle des débits dans le fleuve (Jordan et al., 2007).

Les aménagements hydroélectriques à accumulation ont la possibilité de stocker un certain volume d'eau durant les crues grâce au volume libre du réservoir. Le volume stocké n'alimentera donc pas le cours d'eau aval pendant la crue. Il convient dès lors de maximiser le volume de stockage au moment opportun pour minimiser le débit d'écoulement. Par ailleurs, tout volume d'eau lâché vers l'aval par saturation ou fermeture des captages, par dépassement des capacités de pompage, par déversement sur le couronnement ou par les vannes de vidange rejoindra immanquablement le cours d'eau aval, éventuellement au plus mauvais moment. Il convient donc de minimiser les lâchers d'eau, en particulier pendant la pointe de la crue. Par extension, il est nécessaire pour maximiser l'effet de laminage produit par les retenues d'accumulation, que ces dernières ne se trouvent pas en situation d'application du règlement d'urgence. En effet, le règlement d'urgence s'applique lorsque le niveau de la retenue augmente dangereusement et risque de compromettre la sécurité de l'ouvrage de rétention. Il donne des recommandations impératives qui nécessitent d'arrêter les pompages, de fermer les captages, de turbiner et finalement d'ouvrir les vannes de vidange. Ces manœuvres ont pour effet des lâchers d'eau pendant la pointe de la crue en différents endroits du réseau tels les stations de pompage, les captages, les restitutions de turbines et la digue elle-même. Par une opération préventive, il devient toutefois possible de libérer un volume de rétention avant la crue pour l'utiliser pendant. Cette opération est totalement réussie si, à la fin de la crue, le niveau de la retenue est aussi haut que possible sans avoir conduit à l'application $\mathrm{du}$ règlement d'urgence de l'aménagement.

Finalement, rappelons que l'objectif de l'optimisation est la minimisation du coût des dégâts dus à la crue. Ceci peut être obtenu par la réduction du débit de pointe de la crue, précisément grâce au laminage des crues par les aménagements hydroélectriques. Dans le cas d'une capacité de stockage probablement insuffisante, le turbinage et/ou la vidange préventifs peuvent être effectués, mais ils doivent impérativement s'arrêter avant la pointe de l'hydrogramme de crue afin de ne pas augmenter le débit de pointe à ce moment crucial.

Ces grands principes peuvent être considérés comme des objectifs partiels de l'optimisation, ils se résument comme suit :

- maximiser l'effet de laminage et donc le volume capté

- minimiser les lâchers d'eau

- en cas de capacité de stockage insuffisante, effectuer des opérations préventives (turbinage et/ou vidange) avant la pointe de la crue 
- effectuer les opérations préventives de manière à ne pas être contraint d'appliquer le règlement d'urgence

- réaliser l'opération la plus économique possible

- objectif final de l'optimisation : minimiser les coûts des dégâts en réduisant le débit de pointe de crue

- Avant de détailler la méthode d'optimisation, les principales contraintes du problème d'optimisation sont rappelées ici :

- limites physiques des ouvrages de rétention

- caractéristiques techniques des ouvrages (prises d'eau, collecteurs, turbines, pompes, ouvrages d'évacuation)

- règlements d'urgence

- caractéristiques de l'écoulement dans les cours d'eau

- topologie du réseau hydrographique incluant les aménagements hydroélectriques

Les variables d'optimisation sont les moyens qui permettent de réaliser les objectifs de réduction des dégâts dans le bassin versant par rétention de volumes d'eau dans les retenues existantes. Ces moyens concernent dont uniquement le contrôle du volume de stockage et se limitent aux turbines et aux vidanges de fond des retenues. Cela signifie que pour contrôler le niveau de la retenue, les horaires de fonctionnement des turbines, les horaires d'ouverture et de fermeture des vannes de vidange peuvent être calculés, tout comme les durées de fonctionnement des prises d'eau sur des bassins versants voisins ainsi que le pompage. Dans ce modèle, les variables liées à l'utilisation des prises d'eau n'ont pas été considérées, car la fiabilité des ouvrages lors des crues et leur automatisation n'est pas suffisante pour être exploitée en situation réelle. Enfin, le début du turbinage préventif n'a pas été varié et a été fixé à l'instant présent, car en situation d'urgence il n'y a plus de temps à perdre et l'attente avant de vider la retenue n'est jamais optimale.

\section{IV.2 FONCTIONNEMENT DE L'OPTIMISATION}

Le fonctionnement du modèle d'optimisation se divise en deux phases qui suivent le calcul des prévisions hydrologiques. La première phase est appelée optimisation locale et consiste à analyser les apports prévus dans une retenue pour en déduire les rejets nécessaires. L'objectif est ici de déterminer la vidange préventive nécessaire permettant de stocker tous les apports potentiels sans faire déborder la retenue pendant la crue. L'objectif est qu'à la fin de la crue le niveau de la retenue soit à son maximum admissible. Ce calcul est effectué en suivant les principes énoncés au chapitre précédant par une évaluation des volumes entrants fournis par le modèle de prévision hydrologique, et des volumes sortants, calculés par résolution d'équations de bilan (Jordan, 2007). Chacun des aménagements hydroélectriques est donc analysé lors de l'optimisation locale, sans considération pour les autres aménagements inclus dans le modèle. Les données nécessaires au calcul de l'optimisation locale sont les débits entrants dans la retenue, son niveau initial ainsi que l'indication du fonctionnement des ouvrages de captage et de pompage.

La deuxième phase est appelée optimisation globale et doit trouver les opérations préventives optimales pour le système complet incluant tous les aménagements hydroé- lectriques. Pour réaliser ce calcul, des fonctions de coût ont été intégrées dans le modèle. Les coûts considérés sont premièrement les coûts des débordements dans le cours d'eau principal en différents points de contrôle. Ces coûts sont élevés dans le modèle et conditionnent fortement la solution optimale. Le modèle cherchera ainsi par tous les moyens à limiter le débit dans le cours d'eau principal à des valeurs inférieures aux capacités du cours d'eau. Deuxièmement, les coûts des débordements dans les affluents situés à l'aval des ouvrages d'évacuation (vannes de fond et évacuateurs de crue) sont activés en général si l'un des ouvrage d'évacuation fonctionne avec un débit trop important. Ces coûts permettent de considérer l'effet défavorable de forts débits pour le cours d'eau situé à l'aval d'un barrage. Troisièmement, les coûts des volumes d'eau perdus car évacués (par la vanne de vidange ou par un organe d'évacuation de surface) sont calculés afin de limiter l'utilisation des ouvrages d'évacuation du débit. Enfin, les coûts liés au manque à gagner de production hydroélectrique (en cas d'interdiction de turbiner) ou aux pertes économiques de turbinage (obligation de turbiner à bas prix) sont considérés. Les données nécessaires pour l'optimisation globale sont, en plus des données utilisées lors de l'optimisation locale, les débits prévus sans aménagements aux points de contrôle, les valeurs associées aux fonctions de coût et les capacités des cours d'eau aux points de contrôle. A la fin de cette deuxième phase de l'optimisation, la solution optimale est obtenue : il s'agit, pour chaque aménagement hydroélectrique, des horaires d'utilisation des turbines, des pompes et des vannes de vidange.

\section{IV.3 PERFORMANCE POTENTIELLE DES OPÉRATIONS PRÉVENTIVES EN VALAIS}

Le système de prévision et de gestion des crues en Valais doit permettre de limiter les débits dans le Rhône lors de crues de type centennal. Il est cependant intéressant d'analyser la performance potentielle d'un tel système lors d'événements plus importants afin de déterminer si son utilisation pourrait améliorer la sécurité du bassin versant. Cette analyse a été effectuée sur la base de scénarios tirés des prévisions hydro-météorologiques de MétéoSuisse. En effet, des prévisions du modèle aLMo ont parfois produit des volumes de précipitations si importants qu'ils ont conduit le modèle de prévision de débit à calculer des débits correspondant à des crues de type millénal. Dans ce paragraphe est illustré l'exemple de la prévision du 22 septembre 1993 avec l'influence des retenues alpines sur la réduction des débits de pointe dans le Rhône (fig. 7).

L'exemple présenté ici a été construit de la manière suivante : la prévision hydro-météorologique a d'abord été calculée par le modèle aLMo pour les 72 heures suivant le 22 septembre 1993 à $12 \mathrm{~h}$ (conditions initiales correspondant à la situation réelle du moment, y compris les conditions relatives aux aménagements hydroélectriques). Ces prévisions ont été exploitées avec le logiciel Routing System II (RS2) pour calculer la prévision hydrologique à 72 heures sans retenues (les aménagements hydroélectriques ne sont pas considérés dans le modèle). Une prévision hydrologique 


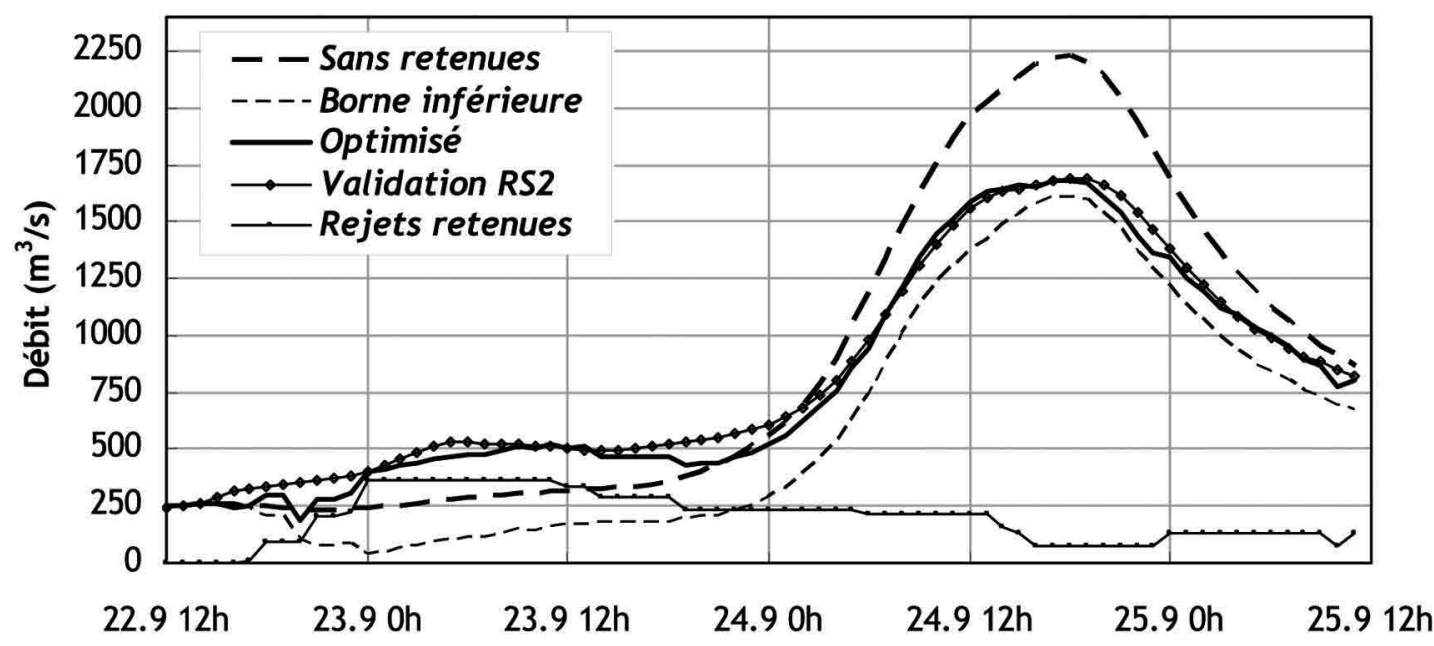

Figure 7 : Crue simulée selon la prévision du 22 septembre 1993 à 12h. Influence des opérations préventives des aménagements hydroélectriques sur le débit de pointe du Rhône à la Porte du Scex.

des débits entrants dans les retenues a également été calculée en tenant compte cette fois-ci des ouvrages hydrauliques existants sur le bassin versant. Ces données ont été transférées au modèle d'optimisation afin de calculer les opérations préventives optimales (hydrogrammes « Optimisé », « Borne inférieure » et « Rejets retenues »). Finalement, les opérations préventives optimales ont été à nouveau transférées dans le modèle de prévision hydrologique RS2 pour validation en tenant compte des retenues ( Validation RS2 »).

La figure 7 montre que le débit de pointe prévu sans retenues aurait atteint $2250 \mathrm{~m}^{3} / \mathrm{s}$ à la Porte du Scex (exutoire du bassin versant du Rhône supérieur). Si toute l'eau arrivant à l'amont des ouvrages de captage avait pu être captée (dans la limite des capacités des ouvrages) et avait pu être stockée (retenues de volume infini), le débit aurait pu être réduit à $1604 \mathrm{~m}^{3} / \mathrm{s}$, soit une réduction de $29 \%$ du débit de pointe («Borne inférieure »). En tenant compte de la situation réelle le 22 septembre 1993 à 12 h et de la capacité des aménagements hydroélectriques à stocker et turbiner ces eaux, le débit de pointe à l'exutoire du bassin versant pouvait être réduit de $25 \%$ à $1682 \mathrm{~m}^{3} / \mathrm{s}$ par des opérations de turbinage et de vidange préventives ("Optimisé »). L'hydrogramme calculé par l'outil d'optimisation est également comparé à l'hydrogramme calculé par le modèle de prévision hydrologique ("Validation RS2 ») qui présente des résultats conformes. Finalement, l'analyse des rejets optimisés (« Rejets retenues ») illustre la stratégie optimale de gestion des crues par les aménagements hydroélectriques. La somme des rejets (turbines et vidanges de fond) atteint $364 \mathrm{~m}^{3} / \mathrm{s} 36$ heures avant la pointe de crue et seulement $74 \mathrm{~m}^{3} / \mathrm{s}$ pendant la pointe. Les retenues profitent donc de la période précédant la crue pour abaisser leur niveau par turbinage, ce qui leur permet de maximiser leur effet de laminage pendant la période critique qui débute dans la matinée du 24 septembre 1993.

Cette analyse montre que malgré des retenues presque complètement pleines 48 heures avant une crue exceptionnelle, il est possible de réduire considérablement le débit de pointe à l'exutoire du bassin versant du Rhône supérieur grâce aux opérations de turbinage et de vidange préventives des retenues. Ainsi, dans ce bassin versant où $30 \%$ de la surface est drainée vers des grandes retenues, une réduction de $25 \%$ du débit de pointe à son exutoire peut être atteinte en effectuant des opérations préventives 48 heures avant une crue de type millénal.

\section{$V \square$ APPLICATION DU SYSTEME MINERVE A UNE SITUATION RELLE}

\section{V.1 PRINCIPE DE L'ANALYSE}

Le système MINERVE fonctionne actuellement sur une base déterministe. Cela signifie qu'une seule prévision hydrologique est disponible pour une échéance de 72 heures, et donc qu'une seule stratégie optimale est calculée à la fois. La procédure en situation de crue est donc la suivante : une fois l'alerte donnée par MétéoSuisse, la première prévision hydrologique est calculée avec considération des conditions initiales du moment (modèle hydrologique, aménagements hydroélectriques). Sur cette base est déterminée la stratégie de gestion des retenues qui est éventuellement appliquée jusqu'à disposition d'une nouvelle prévision hydrologique 12 heures plus tard. La nouvelle stratégie de gestion des retenues est ainsi déterminée et confrontée aux observations sur le terrain pour évaluer la plausibilité des prévisions hydro-météorologiques et hydrologiques. Lorsque la crue est terminée, le processus s'arrête.

Le processus complet a été testé pour les crues d'août 1987, de septembre 1993 et d'octobre 2000. Il a de plus été exploité de manière opérationnelle pour la première fois lors d'une alerte en septembre 2006. Dans ce chapitre est présenté l'exemple de la crue d'octobre 2000, plus important événement de ce type observé sur le bassin versant du Rhône supérieur. 


\section{V.2 PRÉVISIONS HYDROLOGIQUES LORS DE LA CRUE D'OCTOBRE 2000}

Les prévisions hydrologiques calculées par le système MINERVE sont présentées à la figure 8 et sont comparées avec les observations. La grande variabilité des prévisions déterministes y est remarquable. Cela est dû essentiellement à la variabilité des prévision des précipitations du modèle aLMo (Jordan, 2007). Dans ces conditions, il semble délicat d'ordonner des opérations de vidange préventives des retenues hydroélectriques, car des pertes économiques importantes pourraient résulter d'une erreur d'appréciation des prévisions. Cependant, l'exercice a été effectué par le modèle d'aide à la décision du système MINERVE, avec mise à jour de la stratégie de gestion des retenues toutes les 12 heures sur la base des prévisions hydro-météorologiques du modèle aLMo et ensuite toutes les 6 heures avec confrontation aux observations sur le terrain.

\section{V.3 PERFORMANCE OPÉRATIONNELLE DU SYSTÈME MINERVE}

Les hydrogrammes à l'exutoire du bassin versant du Rhône supérieur avec ou sans application du système MINERVE sont maintenant comparés (fig. 9). Tout d'abord, l'hydrogramme sans retenues est présenté, puis les hydrogrammes optimisés (avec prévisions parfaites) et enfin les hydrogrammes opérationnels (avec prévisions réelles du modèle aLMo).

Lors de cet événement, le débit sans retenues aurait atteint $1508 \mathrm{~m}^{3} / \mathrm{s}$, alors que le débit observé a atteint $1358 \mathrm{~m}^{3} / \mathrm{s}$. L'influence observée des aménagements hydroélectrique a dont été une réduction du débit de pointe à l'exutoire du bassin versant de près de $10 \%$. En analysant les rejets observés, on remarque une pointe de ces derniers à $225 \mathrm{~m}^{3} / \mathrm{s}$ simultanément à la pointe de la crue. En clair, il y avait plus de $200 \mathrm{~m}^{3} / \mathrm{s}$ de trop dans le cours d'eau lors de la pointe de la crue.
Si des prévisions parfaites avaient été disponibles, la stratégie de gestion optimale des retenues aurait pu être appliquée. Le débit aurait pu être réduit à $1185 \mathrm{~m}^{3} / \mathrm{s}$ ("Optimisé ») et les rejets limités à $81 \mathrm{~m}^{3} / \mathrm{s}$ ("Rejets optimisés ») pendant la pointe de la crue. Cela correspond donc à une réduction de $21 \%$ du débit de pointe sans retenues.

Finalement, l'application du modèle d'aide à la décision dans des conditions opérationnelles aurait permis également de réduire le débit de pointe de la crue à la Porte du Scex. En effet, l'exploitation déterministe des prévisions hydrométéorologiques du modèle aLMo dans le modèle d'optimisation de la gestion des retenues aurait permis de limiter le débit de pointe à $1202 \mathrm{~m}^{3} / \mathrm{s}$ (« Opérationnel ») et les rejets à $102 \mathrm{~m}^{3} / \mathrm{s}$ («Rejets opérationnels »), ce qui correspond à une diminution de $20 \%$ du débit de pointe du Rhône lors de la crue. Cela correspond également à un doublement de l'effet de laminage protecteur des aménagements hydroélectriques.

Il est intéressant de noter que malgré une forte variabilité des prévisions hydrologiques, des décisions de bonne qualité peuvent être prises sur la base de ce modèle d'aide à la décision. Cela provient de la nature des données utilisées dans l'optimisation. En effet, lorsque les retenues sont pratiquement pleines (ce qui est le cas en fin d'été et en début d'automne), des forts débits entrants nécessitent forcément une vidange préventive et un abaissement du niveau de la retenue. Dans ce contexte, une erreur sur le volume entrant prévu n'aura pas de grands impacts sur la décision d'abaisser le niveau 36 heures avant la crue. Ensuite, la décision d'arrêter les turbines et de fermer les vidanges, si elle est réalisable (les règlements d'urgence faisant foi) peut être basée sur les mesures de débit dans les cours d'eau, qui ne sont pas entachées de telles erreurs. La stratégie optimale n'est donc pas appliquée dans ces conditions, mais une stratégie très proche de l'optimum peut toutefois être déterminée, comme le démontre l'exemple présenté ici. Les applications aux

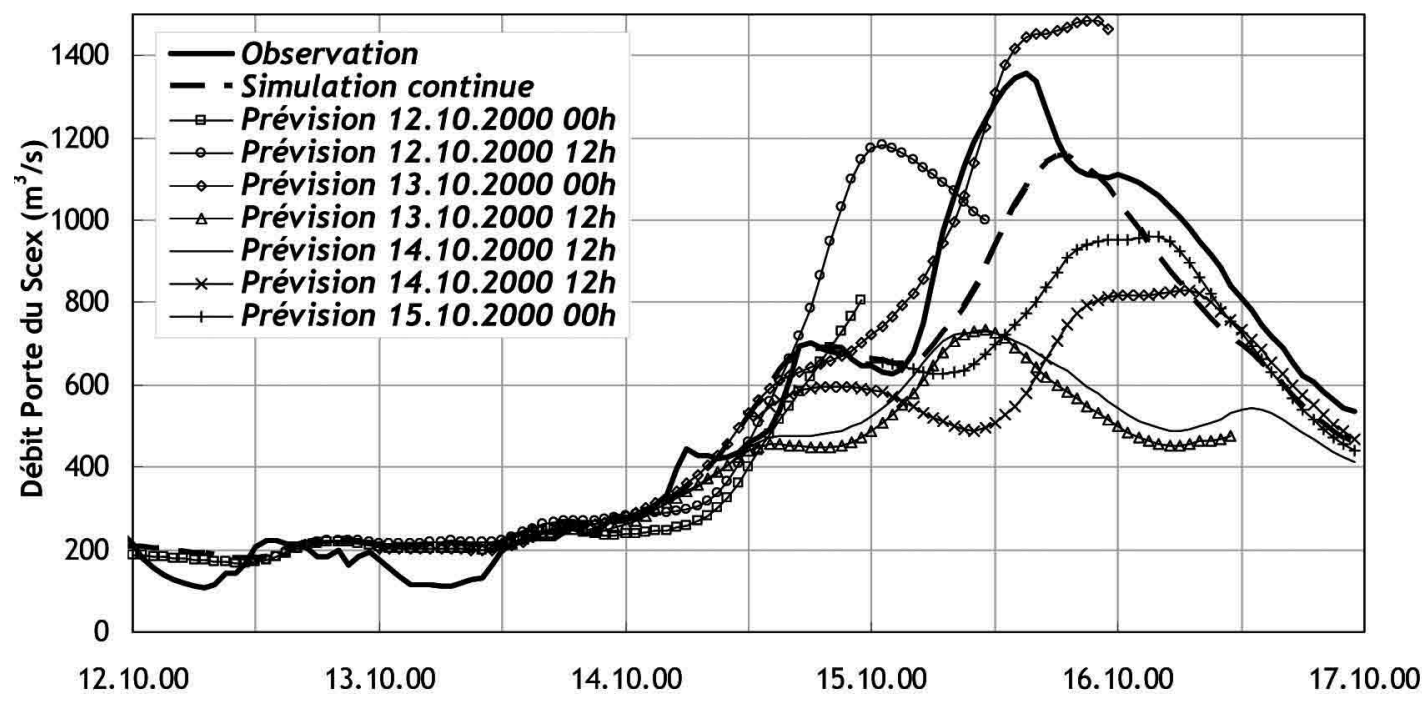

Figure 8 : Prévisions hydrologiques à la Porte du Scex (exutoire du bassin versant du Rhône supérieur) lors de la crue d'octobre 2000. 


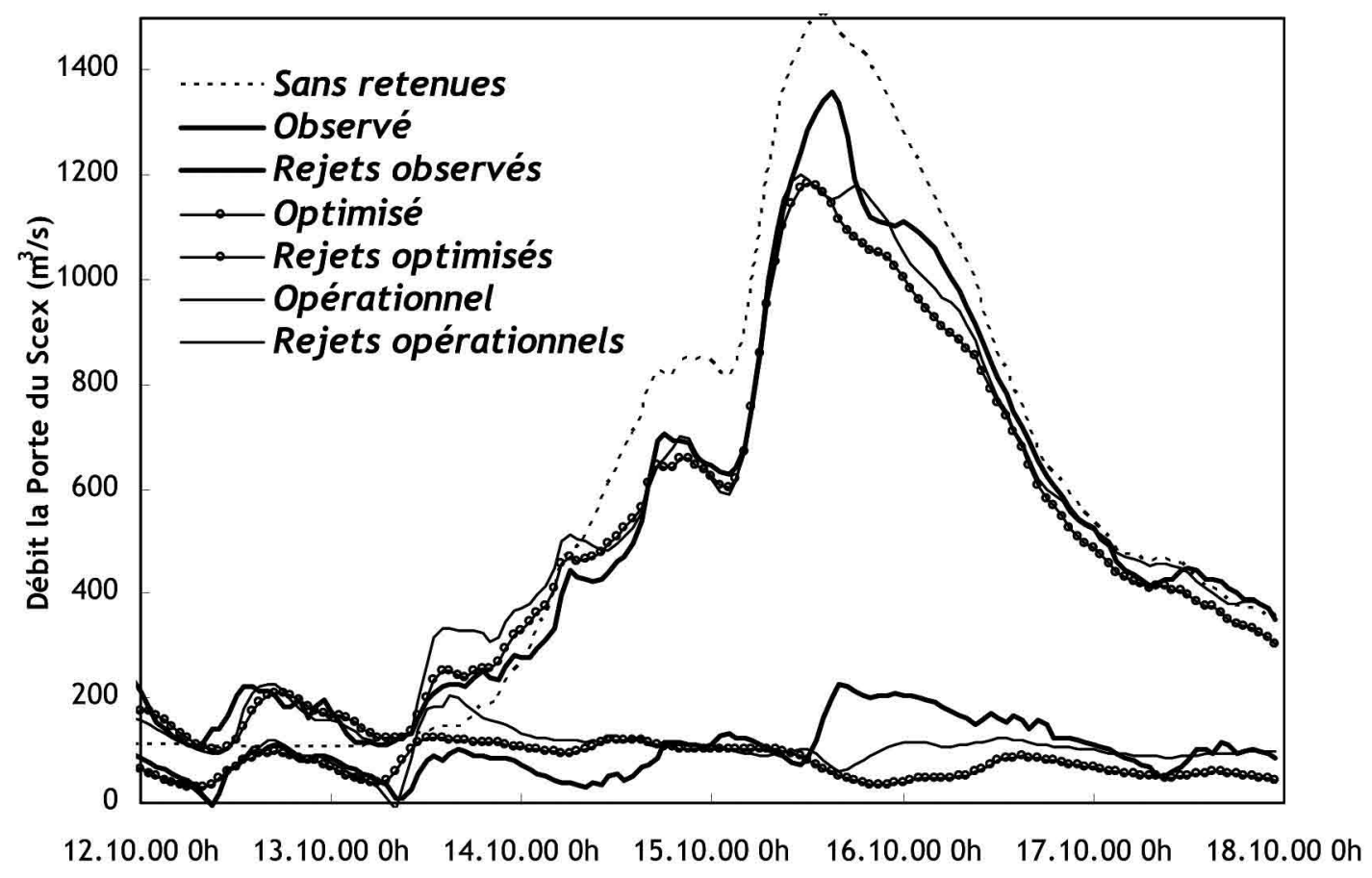

Figure 9 : Hydrogrammes à la Porte du Scex lors de la crue d'octobre 2000.

deux autres crues de 1987 et 1993 ont également fourni des performances similaires (Jordan, 2007).

\section{VI $\square$ CONCLUSIONS}

Le système MINERVE permet de prévoir et gérer les crues, en intégrant les principales composantes hydrologiques et hydrauliques du bassin versant du Rhône à l'amont du Léman. La surface de la région à modéliser, d'environ $5500 \mathrm{~km}^{2}$, confère au bassin versant des temps de réponse de l'ordre de 24 à 36 heures. En raison de sa morphologie alpine, les processus hydrologiques sont très différenciés selon les sous-bassins versants. Enfin, le bassin versant comprend de nombreux aménagements hydroélectriques à accumulation dont les caractéristiques permettent aux exploitants d'influencer considérablement les écoulements dans le réseau hydrographique. Le concept de modélisation semi-distribuée intègre les différents processus hydrologiques et permet l'intégration explicite des aménagements hydroélectriques et des cours d'eau, tout comme la prise en compte de la répartition spatiale des variables météorologiques. Il permet de calculer les débits en tout point du bassin versant du Rhône à l'amont du Léman. Il offre une performance remarquable, compte tenu de la complexité du bassin versant : les indicateurs de Nash varient entre 0.85 et 0.70 de l'amont à l'aval du Rhône, et le bilan hydrique est équilibré. Tous les principaux cycles hydrologiques et de production hydroélectrique sont modélisés, de même que les crues récentes qui ont pu être reproduites de manière très satisfaisante. Le modèle est de plus capable d'assimiler en temps réel les observations, grâce au développement d'une procédure automatique de mise à jour des variables d'état.

Un outil d'optimisation des opérations de vidange préventive des retenues hydroélectriques a été développé dans ce travail. Considérant les 10 principaux aménagements hydroélectriques valaisans, il permet de déterminer, sur la base d'une prévision hydrologique, les durées optimales de turbinage et d'ouverture des vannes des vidanges de fond des barrages. La performance de la gestion optimisée des aménagements hydroélectriques à accumulation lors des crues a de plus été démontrée. En effet, dans le bassin versant du Rhône à l'amont du Léman, des réductions des débits de pointe sans aménagements de 25-30\% peuvent être attendues.

Si le système fonctionne actuellement sur une base déterministe, il est maintenant prévu de le développer pour lui permettre d'intégrer une composante probabiliste. Pour atteindre cet objectif, les prévisions hydro-météorologiques du modèle COSMO-LEPS seront exploitées dans le modèle de prévision hydrologique, mais également dans le modèle d'aide à la décision, afin de permettre au décideur de disposer d'un indicateur de risque associé à chaque décision de gestion des aménagements hydroélectriques.

\section{VII — REMERCIEMENTS}

Le projet MINERVE est financé par l'Etat du Valais et l'Office Fédéral de l'Environnement. Les données météo sont fournies par MétéoSuisse qui participe également à ce projet. 


\section{VIII — RÉFÉRENCES}

[1] Boillat J.-L. (2005) — L'influence des retenues valaisannes sur les crues - Le projet MINERVE, Communication LCH $\mathrm{n}^{\circ} 21$. Proc. Conférence sur la recherche appliquée en relation avec la $3^{e}$ Correction du Rhône - Nouveaux développements dans la gestion des crues, Martigny, Ed. A. Schleiss. 87-101

[2] Consuegra D., Niggli M., Musy A. (1998) - Concepts méthodologiques pour le calcul des crues - Application au bassin versant supérieur du Rhône. Wasser Energie Luft - Eau Energie Air. 9/10 223-231

[3] Garcia Hernandez J., Jordan F., Dubois J., Boillat J.-L. (2008) - Routing System II, modélisation d'écoulements dans des systèmes hydrauliques. Communication $L C H, E d$. A. Schleiss. $3270 \mathrm{pp}$

[4] Hamdi Y., Hingray B., Musy A. (2005) - Un modèle de prévision hydro-météorologique pour les crues du Rhône supérieur en Suisse. Wasser Energie Luft - Eau Energie Air. 11/12 325-332

[5] JORDAN F. (2005) — Gestion des crues par opérations préventives sur les aménagements hydroélectriques à accumulation. Wasser Energie Luft - Eau Energie Air. 11/12 333-337

[6] Jordan F., Boillat J.-L., Dubois J., Schleiss A. (2006) Prévision et gestion des crues par opérations préventives sur les retenues alpines,. Proc XXII Congrès des Grands Barrages, CIGB/ICOLD, Barcelone. 497-510
[7] JORDAN F. (2007) - Modèle de prévision et de gestion des crues - optimisation des opérations des aménagements hydroélectriques à accumulation pour la réduction des débits de crue, thèse de doctorat $n^{\circ} 3711$, Ecole Polytechnique Fédérale, Lausanne. $261 \mathrm{pp}$

[8] Jordan F., Boillat J.-L., Dubois J., Schleiss A. (2007) Optimal operation of multiple storage hydropower schemes for flood management. J. Wat. Resour. Plan. Manag., soumis.

[9] Kaufmann P., Schubiger F., Binder P. (2003) - Precipitation forecasting by a mesoscale numerical weather prediction (NWP) model : eight years of experience. Hydrology and Earth System Sciences. 7(6) 812-832

[10] Nash J. E., Sutcliffe J. V. (1970) - River flow forecasting through conceptual models, part 1 - A discussion of principles. J. of Hydrol. 10(3) 282-290

[11] Raboud P.-B., Dubois J., Boillat J.-L., Costa S., Pitteloud P.-Y. (2001) - Projet MINERVE-Modélisation de la contribution des bassins d'accumulation lors des crues en Valais. Wasser Energie Luft - Eau Energie Air. 11/12 313-317

[12] Schaefli B., Hingray B., Niggli M., Musy A. (2005) - A conceptual glacio-hydrological model for high mountainous catchments. Hydrol. Earth Syst. Sc. Disc. 2 73-117

[13] Shepard D. (1968) - A two-dimensional interpolation function for irregularly-spaced data. Proc., 23 th Conf. ACM. $517-$ $524[1]$ 